\title{
Photocatalytic Activity of the Oxide Layer Formed on NiTi Surface through Thermal Oxidation Process
}

\author{
Kouta Sakamoto*1, Kento Yokoi*1, Aki Saito*2 and Naofumi Ohtsu*3 \\ Instrumental Analysis Center, Kitami Institute of Technology, Kitami 090-8507, Japan
}

In the present study, we attempted to prepare a photocatalytic titanium dioxide $\left(\mathrm{TiO}_{2}\right)$ layer on nickel-titanium (NiTi) alloy surface through a simple thermal oxidation process in air. At $723 \mathrm{~K}$, an amorphous $\mathrm{TiO}_{2}$ layer including a slight amount of Ni was formed on the surface. Above $873 \mathrm{~K}$, the $\mathrm{TiO}_{2}$ layer crystallized into a rutile phase. At $1023 \mathrm{~K}$, a complex oxide of $\mathrm{NiTiO}_{3}$ was also formed. The methylene blue degradation test for evaluating photocatalytic activity showed that the formed $\mathrm{TiO}_{2}$ layers act as photocatalyst under ultraviolet (UV) light illumination, and its activity is superior to that of the surface layer formed by oxidizing a pure Ti substrate. The adhesion strength of the surface layers formed at $723 \mathrm{~K}$ on NiTi alloy was higher than that of a commercially available $\mathrm{TiO}_{2}$-coating. We conclude that thermal oxidation as a surface modification technique is expected to make NiTi alloys give photocatalytic activity. [doi:10.2320/matertrans.M2014114]

(Received March 31, 2014; Accepted May 14, 2014; Published July 25, 2014)

Keywords: nickel titanium, thermal oxidation, photocatalytic activity

\section{Introduction}

A nickel-titanium (NiTi) alloy has been used as a material for dental and medical devices such as root canal files and catheters, due to its shape memory property and superelasticity. ${ }^{1)}$ In medical practice, these devices must generally be sterilized in order to remove microorganisms attached on their surfaces, and high-pressure steam or dry-heat treatments have been used as sterilization processes. However, the risk of infectious disease transmission from the devices persists, even after the aforementioned processes. It is considered that this risk can be reduced if the NiTi alloy itself possesses an antimicrobial activity. Thus far, researchers have made efforts to increase the antimicrobial activity of metallic substrates. $^{2-6)}$

Our research group has focused on photocatalytic $\mathrm{TiO}_{2}$ as a possible antimicrobial medium. Photocatalytic $\mathrm{TiO}_{2}$ has excellent antimicrobial activity under ultraviolet light (UV) illumination, and is not modified in the process. ${ }^{7-10}$ ) Furthermore, $\mathrm{TiO}_{2}$ is a chemically stable, nontoxic material. To coat $\mathrm{TiO}_{2}$ on metallic substrates, chemical and physical deposition techniques such as plasma spraying and sputtering have been used. ${ }^{11,12)}$ However, the adhesion strength of the coating using these techniques is comparatively low, in general. Thus, these processes are not considered suitable for medical devices. Compared to those deposition techniques, the adhesion strength of surface layers formed by oxidizing a metallic surface is high due to its gradual interfacial structure. Anodic oxidation process is possible candidate to prepare $\mathrm{TiO}_{2}$ layers. Wong et al. reported that the oxide layer could be formed on NiTi surfaces through alternating current anodization, resulting in a loose surface layer with large cracks. ${ }^{13)}$ Such loose surface layer may injure a human body, causing inflammation. Therefore, this technique is not suitable for medical devices.

A simple thermal oxidation process in an atmosphere including oxygen is suggested to be an alternative technique

\footnotetext{
${ }^{* 1}$ Graduate Student, Kitami Institute of Technology

${ }^{* 2}$ Undergraduate Student, Kitami Institute of Technology

${ }^{* 3}$ Corresponding author, E-mail: nohtsu@mail.kitami-it.ac.jp
}

for forming $\mathrm{TiO}_{2}$ layers on these alloys. It is well known that the oxidation reaction's elemental selectivity in the thermal oxidation of an alloy is dominated by the standard free energy for the formation of metallic oxides. For instance, the values at $298 \mathrm{~K}$ for $\mathrm{TiO}_{2}$ and $\mathrm{NiO}$ are 889.5 and $211.7 \mathrm{~kJ} \mathrm{~mol}^{-1}$, respectively. This selectivity implies that the Ti contained within the alloy is preferentially oxidized when heated it in air, resulting in the formation of a $\mathrm{TiO}_{2}$ layer. Vojtěch et al. reported that a $\mathrm{TiO}_{2}$ layer was formed on NiTi alloy by heating it in air at $803 \mathrm{~K},{ }^{14)}$ and $\mathrm{Wu}$ et al. reported that a $\mathrm{TiO}_{2}$ layer was prepared on NiTi alloy by heating it in air at $723 \mathrm{~K}^{15)}$ These reports revealed that $\mathrm{TiO}_{2}$ layers could be formed through heating. However, in those studies, the purpose of the formation of $\mathrm{TiO}_{2}$ layers was to create brier for $\mathrm{Ni}$ ion diffusion to the human body, and the photocatalytic activity of the layers was hardly discussed. In this study, therefore, oxide layers were formed on NiTi surfaces through a thermal oxidation process, and their characteristics were investigated in detail. Furthermore, the photocatalytic activities of surface layer were estimated by using a methylene blue (MB) degradation test.

\section{Material and Methods}

NiTi disks (55.07 at\% Ni, NEC TOKIN, Japan), $15 \mathrm{~mm}$ in diameter and $2 \mathrm{~mm}$ in thickness, was used as a substrate. The substrate was mechanically polished using emery papers up to $\# 1200$, and the disk was then ultrasonically rinsed in ethanol for $600 \mathrm{~s}$. The thermal oxidization was conducted using a conventional electronic furnace (FO-100, Yamato Scientific, Japan) in an ambient atmosphere. The substrate was placed in the furnace, heated at oxidizing temperatures ranging from 573 to $1023 \mathrm{~K}$ for $18 \mathrm{ks}$. Then, the furnace was turned off and allowed to cool to room temperature, with the sample inside.

The surface morphologies of the oxidized substrates were observed using scanning electron microscopy (SEM; JCM5000 Neo Scope, JEOL, Japan), with an acceleration voltage of $10 \mathrm{kV}$, using secondary electron image. The crystallinity was investigated using X-ray diffractometry (XRD; New D8 ADVANCE, Bruker AXS, Germany) in the Bragg-Brentano 
geometry using $\mathrm{Cu} \mathrm{K} \alpha$ radiation. The chemical state and depth profile of the surface layers were characterized using X-ray photoelectron spectroscopy (XPS; PHI5000 VersaProbe, ULVAC-PHI, Japan) with monochromatized $\mathrm{Al} \mathrm{K} \alpha$ radiation $(\mathrm{h} v=1486.6 \mathrm{eV})$. For the XPS measurements, the diameter of the X-ray probe was approximately $100 \mathrm{~nm}$, and the photoelectron take-off angle was set at $45^{\circ}$. The binding energy was corrected using the $\mathrm{C} 1 \mathrm{~s}$ peak corresponding to surface adsorbed hydrocarbon $(284.8 \mathrm{eV})$. Elemental depth profiling was done using an Ar ion gun with an acceleration voltage of $4 \mathrm{kV}$. The etching rate estimated from the $\mathrm{SiO}_{2}$ layer was approximately $0.2 \mathrm{~nm} \mathrm{~s}^{-1}$. The adhesion strength of the surface layers on the substrate was estimated from the critical load to exfoliate the oxide layer using nanolayer scratch tester (CSR-2000, Rhesca, Japan). The surface layers were scratched using a diamond stylus with $200 \mathrm{~g} \mathrm{~mm}^{-1}$ spring constant and tip radii of $5 \mu \mathrm{m}$. The lateral rate, amplitude and loading rate were $10 \mu \mathrm{ms}^{-1}$, $100 \mu \mathrm{m}$ and $2.50 \mathrm{mN} \mathrm{s}^{-1}$, respectively.

The photocatalytic activity of the oxidized substrate was estimated using a methylene blue (MB) degradation test. Prior to the test, the specimens were immersed in a polypropylene vessel containing $5 \mathrm{dm}^{3}$ of $10 \mu \mathrm{mol} \mathrm{cm} \mathrm{cm}^{-3} \mathrm{MB}$ aqueous solution, after which the vessels were placed in the dark for $86.4 \mathrm{ks}$, in order to complete the adsorption of MB molecules. The aqueous solution was thereafter refreshed, and irradiated with light of a $370 \mathrm{~nm}$ wavelength and an intensity of $1 \mathrm{~mW} \mathrm{~cm}^{-2}$ for $10.8 \mathrm{ks}$, using an LED. The MB concentration was evaluated every $1.2 \mathrm{ks}$ during the illumination by measuring the absorbance of $\mathrm{MB}$ at $664 \mathrm{~nm}$, using a UVvis spectrometer (UV-2400 PC, Shimadzu, Japan). The evaluated concentration was plotted versus the illumination periods, and $\mathrm{MB}$ degradation rates $\left(\mu \mathrm{mol} \mathrm{cm} \mathrm{cm}^{-3} \mathrm{~s}^{-1}\right)$ were calculated from the slope of the resulting line.

\section{Results and Discussion}

\subsection{Characteristics of the oxidized NiTi surface}

Morphological images of thermally oxidized NiTi alloy surface with various temperatures, observed by SEM, are shown in Fig. 1. An SEM image of an untreated NiTi alloy surface is also shown for comparison. The surface morphology of the alloys oxidized at $573 \mathrm{~K}$ (Fig. 1(b)) and $723 \mathrm{~K}$ (Fig. 1(c)) are similar to that of the untreated alloy surface (Fig. 1(a)). The formation of the surface layer could not be confirmed from the SEM images. On the other hands, a transition in surface morphology, towards higher granularity (Fig. 1(d)), was observed at oxidation temperatures above $873 \mathrm{~K}$, and the exfoliation of specific areas was observed on the surface after oxidizing at $1023 \mathrm{~K}$ (Fig. 1(e)). The grains observed on the surface after oxidizing at $1023 \mathrm{~K}$ were much larger than those observed after oxidizing at $873 \mathrm{~K}$.

The XPS spectra of the Ti 2 , O $1 \mathrm{~s}$ and Ni $2 p$ regions, obtained from NiTi substrates oxidized at 723 and $873 \mathrm{~K}$, are shown in Fig. 2. In both temperatures, the binding energy of the $\mathrm{Ni} 2 \mathrm{p}_{3 / 2}$ peak is $856.2 \mathrm{eV}$ and small satellite peaks associated with Ni $2 p$ peaks were observed. These characteristics in Ni $2 p$ region agree with those of NiO. ${ }^{16)}$ Similarly, in the case of $\mathrm{O} 1 \mathrm{~s}$, the spectral shape for $723 \mathrm{~K}$ almost coincides with that for $873 \mathrm{~K}$. The binding energy of the $\mathrm{O} 1 \mathrm{~s}$

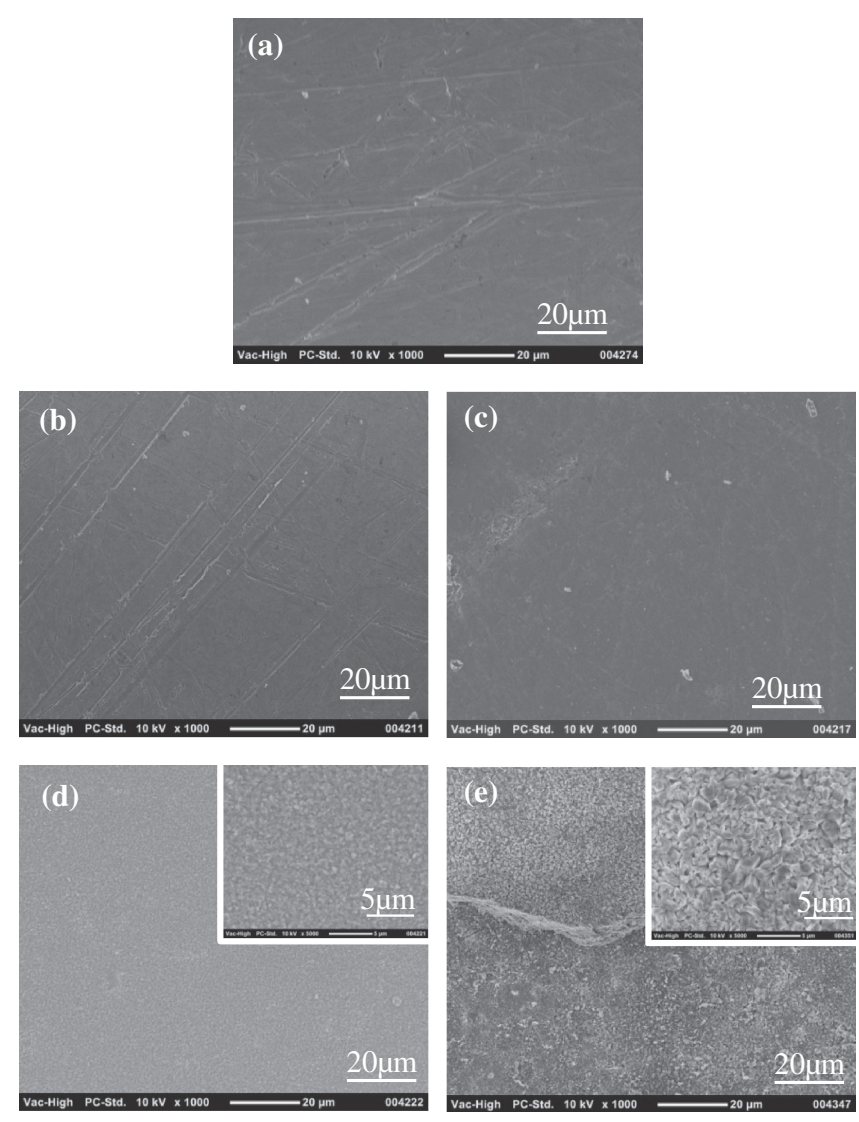

Fig. 1 SEM images of NiTi alloy substrate without treatment (a), and with thermal oxidization at temperatures of $573 \mathrm{~K}$ (b), $723 \mathrm{~K}$ (c), $873 \mathrm{~K}$ (d), and $1023 \mathrm{~K}(\mathrm{e})$

peak is $530.2 \mathrm{eV}$, which agrees with that for metallic oxide state. ${ }^{16)}$ On the other hand, slight chemical shift in Ti $2 p$ spectra was observed between the 723 and $873 \mathrm{~K}$ surfaces. The binding energy of the Ti $2 \mathrm{p}_{3 / 2}$ peak for $873 \mathrm{~K}$ is $458.5 \mathrm{eV}$, which almost agrees with that of $\mathrm{TiO}_{2},{ }^{16}$ ) whereas the energy for $723 \mathrm{~K}$ is slightly lower than that for $873 \mathrm{~K}$. The atomic ratio of $\mathrm{Ni}$ to $\mathrm{Ti}([\mathrm{Ni}] /[\mathrm{Ti}])$ for 723 and $873 \mathrm{~K}$, calculated from the spectral intensity of $\mathrm{Ni} 2 \mathrm{p}$ and $\mathrm{Ti} 2 \mathrm{p}$, were 1.1 and 0.4 , respectively. Wu et al. analyzed the binding energy of nanostructured Ti-Ni-O compounds with various $[\mathrm{Ni}] /[\mathrm{Ti}]$ ratios using XPS, revealed that the energy of Ti $2 p_{3 / 2}$ was shifted toward low value with the increase of $[\mathrm{Ni}] /[\mathrm{Ti}]$ ratio. ${ }^{17)}$ Considering from their report, we concluded that the slight shift of Ti $2 p$ peaks was originated from the difference in the $[\mathrm{Ni}] /[\mathrm{Ti}]$ ratio in the topmost surface. Combined, these results indicate that the surface chemical state of the oxidized NiTi alloys is composed of $\mathrm{TiO}_{2}$ with small amounts of $\mathrm{NiO}$.

XRD patterns of the oxidized NiTi alloys at various temperatures were measured using a Bragg-Brentano geometry, and are shown in Fig. 3. In the case of oxidation temperatures below $723 \mathrm{~K}$, oxides peaks were hardly observed. After oxidizing at $873 \mathrm{~K}$, peaks corresponding to the rutile phase of $\mathrm{TiO}_{2}$ appeared in the patterns. For oxidizing temperatures between 873 and $1023 \mathrm{~K}$, the intensities of the $\mathrm{TiO}_{2}$ peaks drastically increased. This change indicates the extensive development of the oxide layer. Furthermore, small peaks corresponding to the 

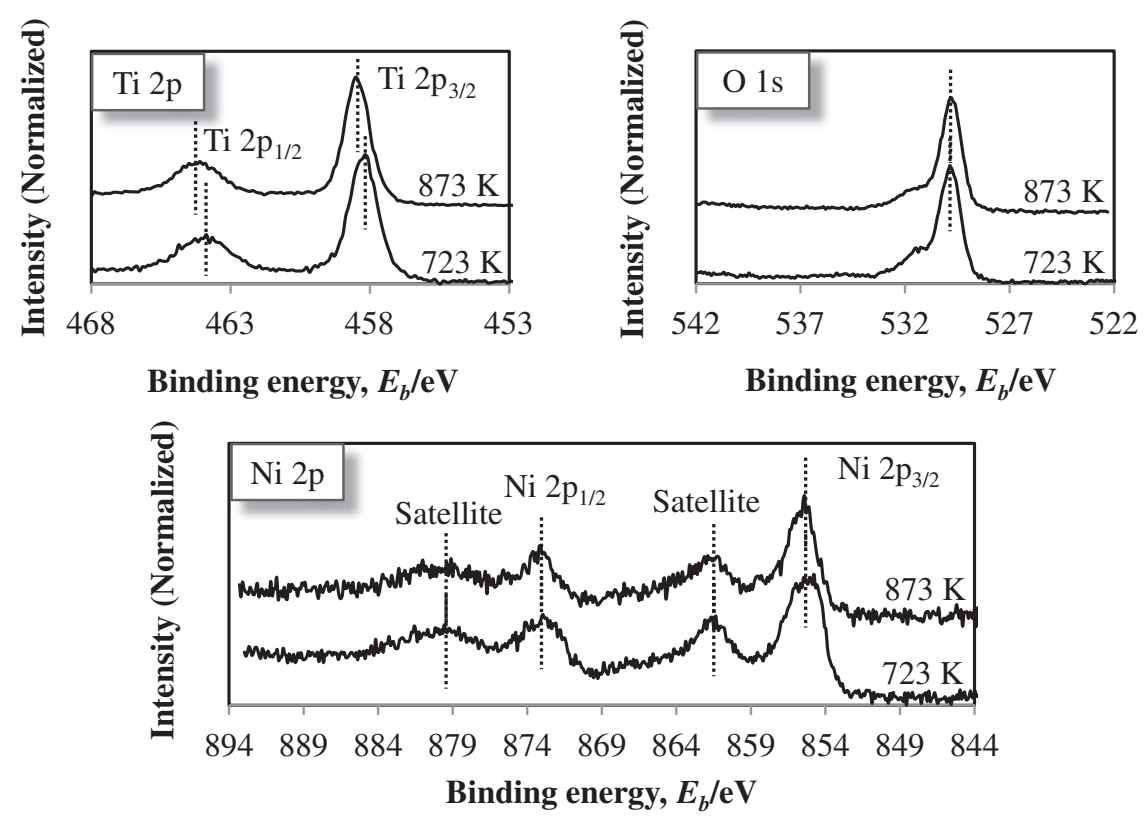

Fig. 2 XPS spectra of Ti 2p, O 1s and Ni 2p regions obtained from the NiTi surface oxidized at 723 and $873 \mathrm{~K}$.

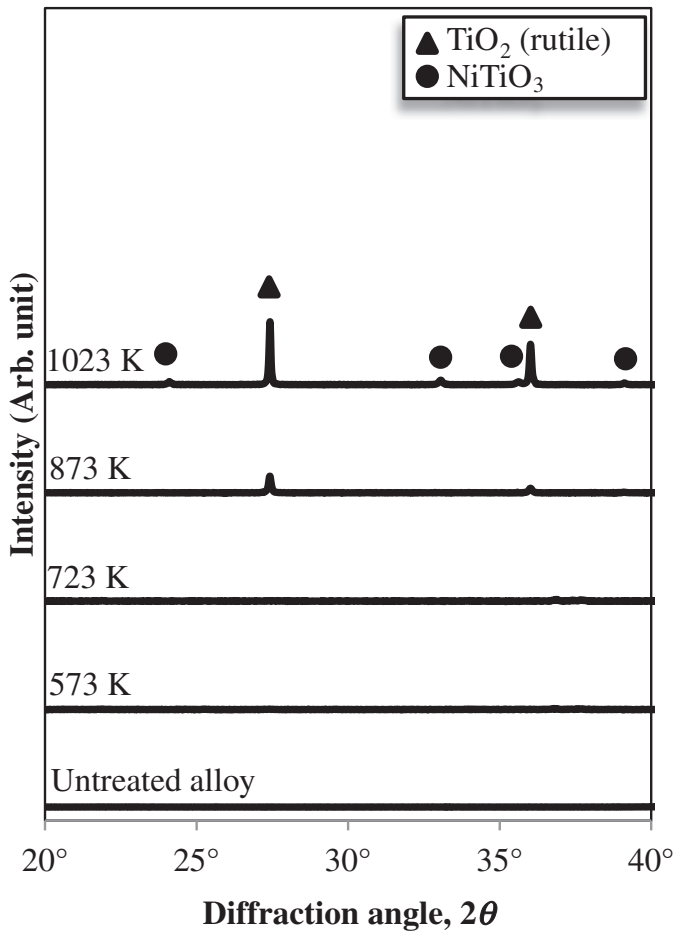

Fig. 3 XRD patterns of the oxidized NiTi alloy substrate measured by using Bragg-Brentano geometry.

complex oxide $\mathrm{NiTiO}_{3}$ were also found in the pattern after oxidation at $1023 \mathrm{~K}$. The XRD results demonstrated that a crystallized $\mathrm{TiO}_{2}$ layer can be formed on NiTi alloys through thermal oxidation by heating above $873 \mathrm{~K}$, although the use of too high of a temperature such as $1023 \mathrm{~K}$, results in the formation of a complex oxide. The $\mathrm{NiTiO}_{3}$ is formed by reacting $\mathrm{NiO}$ and $\mathrm{TiO}_{2}$, and its standard free energy decreases with increasing temperature. ${ }^{18)}$ When employing comparatively low temperature, $\mathrm{NiO}$ formed in surface layer. The $\mathrm{NiO}$ react with $\mathrm{TiO}_{2}$ when raising the temperature, thereby forming $\mathrm{NiTiO}_{3}$.
XRD measurements cannot detect amorphous compounds. Thus, in order to confirm the existence of amorphous $\mathrm{TiO}_{2}$ on the surface, we measured a depth profile of the oxidized NiTi alloy surface. Elemental depth profiles for NiTi alloys oxidized at 573 and $723 \mathrm{~K}$ are shown in Fig. 4. In the case of $573 \mathrm{~K}$, oxygen was detected only in the topmost surface region, and decreased abruptly away from the surface. This result indicates that a distinct $\mathrm{TiO}_{2}$ layer was not formed on the surface. On the other hand, after heating at $723 \mathrm{~K}$, oxygen diffused into the alloy up to about $100 \mathrm{~nm}$ from the surface, and the atomic ratio of $\mathrm{O}$ to Ti was approximately 2 to 1 in this region. Concomitantly, a Ni content of approximately 10 at $\%$ was found in the shallower region. Firstov et al. reported that the inward diffusion of oxygen and the outward diffusion of $\mathrm{Ti}$ from the interior occur simultaneously when oxidizing NiTi alloy at the temperature above $673 \mathrm{~K} .{ }^{19)}$ The similar diffusion of $\mathrm{Ni}$ also occurs during the oxidization; however, its speed is slower than that of Ti, thereby forming Ti-oxide leyer. ${ }^{19)}$ We considered that the Ni remaining in the surface layer diffused toward both outward and inward directions after forming Ti-oxide because $\mathrm{Ni}$ atoms diffuse easily through defective $\mathrm{TiO}_{x}{ }^{19,20)}$ Consequently, the Ni-rich regions at the topmost surface and beneath the oxide layer formed on NiTi alloy.

\subsection{Photocatalytic activity of the oxide layer}

The formation of the crystallized or amorphous $\mathrm{TiO}_{2}$ layer was confirmed on a NiTi alloy oxidized at temperatures above $723 \mathrm{~K}$. This result lets us expect that the oxidized alloy should act as a photocatalyst. The photocatalytic activity under UV light illumination as estimated by the MB degradation test is shown in Fig. 5. Test results obtained using two types of control samples consisting of first, pure $\mathrm{Ti}$ oxidized at various temperatures, and second, a $\mathrm{TiO}_{2}$ layer formed using a commercially available $\mathrm{TiO}_{2}$-coating regent (ST-K211, Ishihara Sangyo, Japan), are also shown in the figure. In the case of the NiTi alloy, the degradation rate of the $\mathrm{MB}$ was almost zero at an oxidation temperature of 

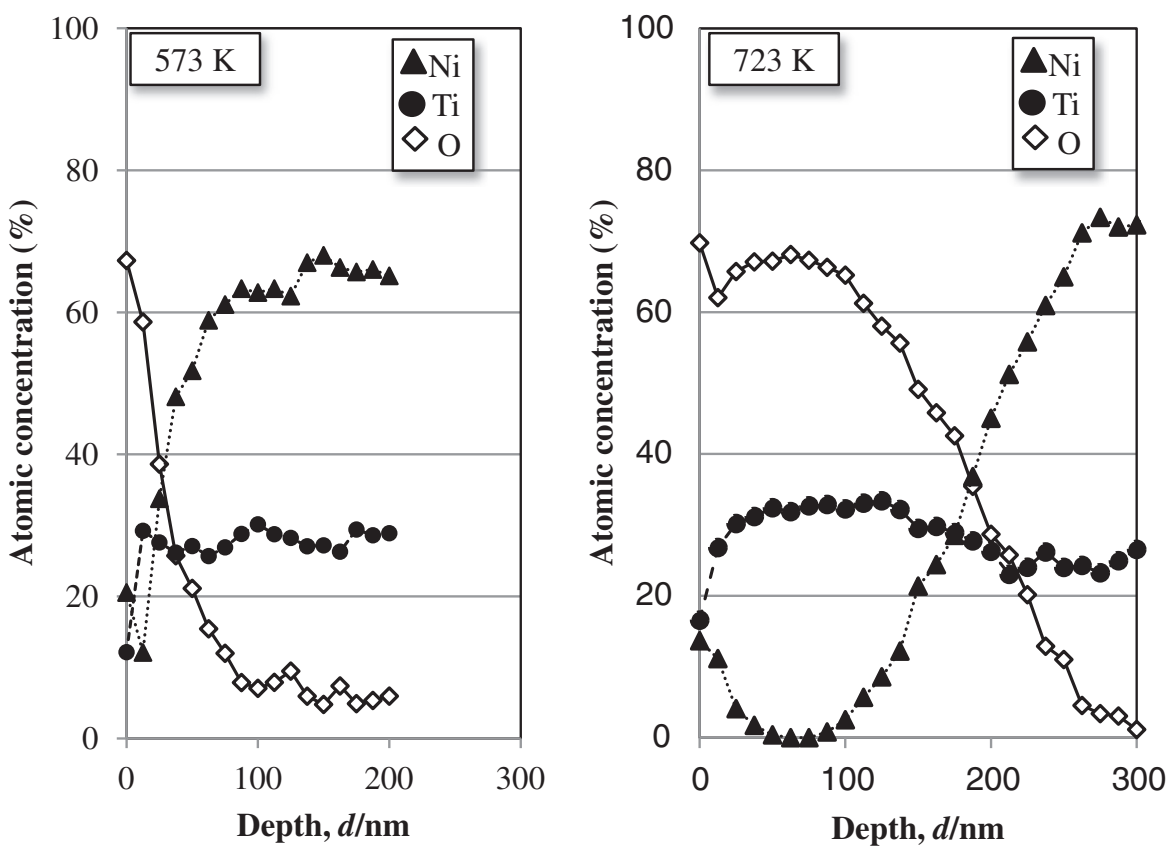

Fig. 4 The elemental depth profiles of the NiTi surface oxidized at 573 and $723 \mathrm{~K}$.

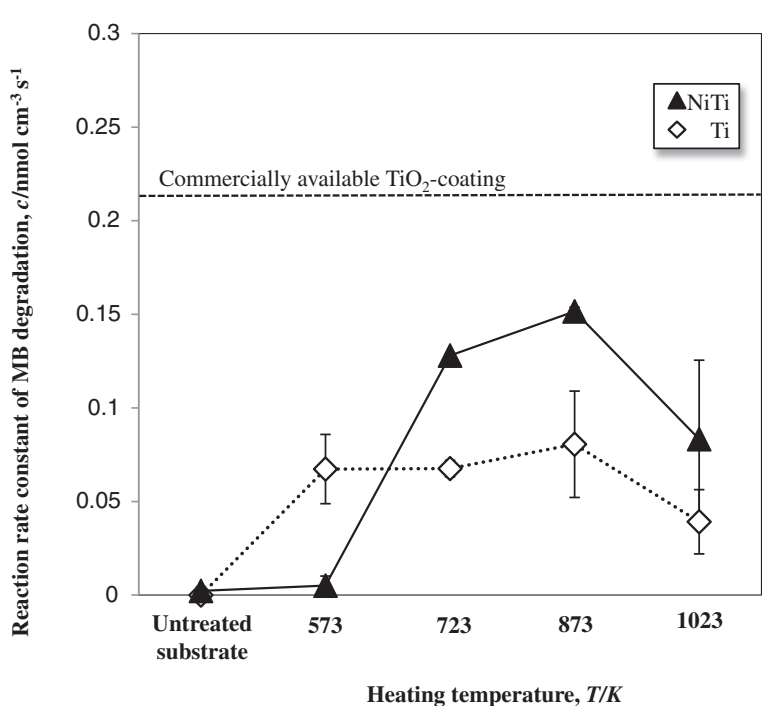

Fig. 5 MB degradation rate of the NiTi alloy substrates oxidized with various temperatures. This result obtained from commercial-regent $\mathrm{TiO}_{2}$ coating is also inserted. All error bars are mean \pm S. D.; $n=3$.

$573 \mathrm{~K}$. The rate drastically increased for oxidation temperatures greater than $723 \mathrm{~K}$, and the highest value was observed for an oxidation temperature of $873 \mathrm{~K}$. The rate decreases if the sample is oxidized above $1023 \mathrm{~K}$. These results show that NiTi alloys oxidized above $723 \mathrm{~K}$ act as a photocatalyst under UV light illumination, although the activities are lower than that of a coating formed using a commercially available regent. It should be noted that the value at $873 \mathrm{~K}$ is slightly higher than that of $723 \mathrm{~K}$. This difference is probably due to the crystallinity of the oxide layer. Furthermore, the usage of too high temperature deteriorates this activity because the peeling off of the layer occurs. $^{21)}$

Photocatalytic activity was also observed on the oxidized pure Ti substrate (Fig. 5). However, for oxidation temperatures above $723 \mathrm{~K}$, the rates are lower than those for oxidized NiTi alloy surfaces. We conjectured that this surprising result originates from the $\mathrm{NiO}$ present on the topmost surface. Sreethawong et al. reported that the loading of $\mathrm{NiO}$ enhances the photocatalytic activity of $\mathrm{TiO}_{2}$ because $\mathrm{Ni}^{2+}$ oxidation state in $\mathrm{NiO}$ can trap photo-generated electrons, thereby suppressing the recombination of the photo-generated electrons-hole pairs. ${ }^{22)}$ The trapped electron is released immediately; therefore, the activity is not deteriorated. Consequently, we consider that the $\mathrm{Ni}^{2+}$ present in the $\mathrm{TiO}_{2}$ layer enhanced the photocatalytic activity of the oxidized NiTi surface. On the other hand, it is well-known that $\mathrm{Ni}$ ion released from a metallic surface causes allergic reaction and cytotoxicity. This effect makes us hesitate to use oxidized NiTi as medical devices, although $\mathrm{NiO}$ in the topmost surface is beneficial in the view from photocatalytic activity. However, the concentration of $\mathrm{Ni}$ existing in the topmost surface was below 20 at\%, which was lower than that of $\mathrm{Ni}$ contained within $\mathrm{NiTi}$ alloy. Furthermore, we consider that a NiTi alloy with photocatalytic activity would be used in medical devices that are repeatedly used clinically, such as root canal files and catheters. In these applications, the amount of $\mathrm{Ni}$ ion released from the surface would be trivial because these devices are not implanted for a long time in a human body. On the basis of these considerations, we concluded that the harmful effect from the $\mathrm{NiO}$ present in topmost surface is negligible.

\subsection{The adhesion strength of the oxide layer on the NiTi}

A merit of thermal oxidization process is the ability to form surface layers having high adhesion strength. To confirm this, we estimated the adhesion strength of the oxide layer on the NiTi alloys using a nanolayer scratch tester. The critical loads of the oxide layer for oxidation temperature of 723 and $873 \mathrm{~K}$ are compared with that of commercial-reagent $\mathrm{TiO}_{2}$-coating in Fig. 6. We couldn't measure the strength of the layer oxidized at $1023 \mathrm{~K}$ because the layer had already exfoliated after the oxidation (Fig. 1(e)). The critical load of 


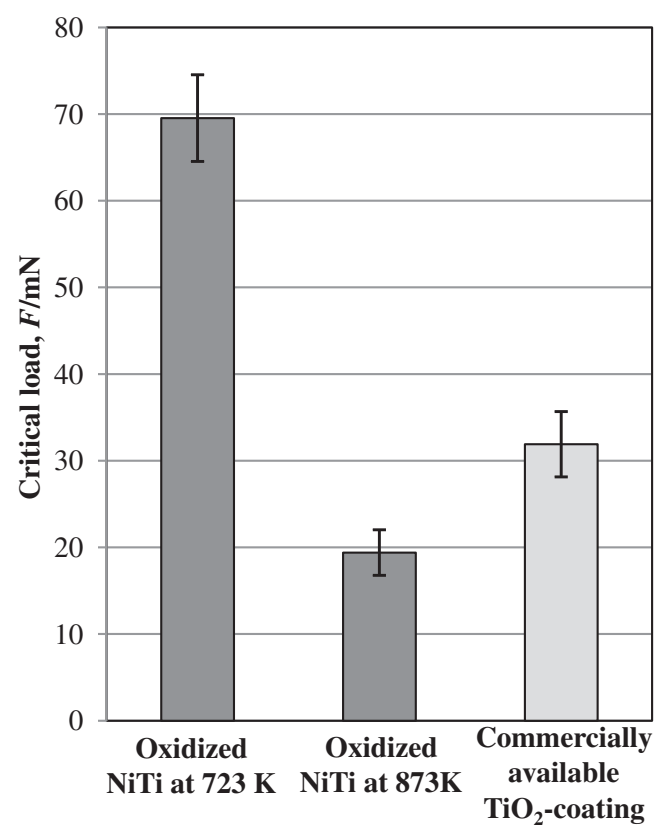

Fig. 6 The critical load of the oxide layer formed by oxidizing NiTi alloy. This result obtained from commercial-regent $\mathrm{TiO}_{2}$-coating is also inserted for comparison. All error bars are mean \pm S. D.; $n=3$.

the layer oxidized at $723 \mathrm{~K}$ is higher than that oxidized at $873 \mathrm{~K}$, which is more than double of the commercial-regent $\mathrm{TiO}_{2}$-coating. From the XPS profile (Fig. 4), the thickness of oxide layer formed at $723 \mathrm{~K}$ is about $100 \mathrm{~nm}$, and the layer thickness probably increases with the increase of the temperature. Kim et al. reported that the oxide layer of $1.2 \mu \mathrm{m}$ in thickness was formed on NiTi alloy when heating it at $873 \mathrm{~K}^{23)}$ We therefore considered that the difference of the adhesion strength depending on the temperature is due to the thickness of the oxide layer. ${ }^{24)}$ The surface layer of the sample oxidized at $723 \mathrm{~K}$ showed a notable advantage in its adhesion strength although its photocatalytic activity was inferior to that of commercial-reagent $\mathrm{TiO}_{2}$-coating (Fig. 6). In medical practice, the peeling off of the surface layer is directly linked to the risk of the failure in medical operation; accordingly, the adhesion strength is the most significant property. Consequently, we believe that thermal oxidation using a temperature of $723 \mathrm{~K}$ is the optimum treatment procedure for medical safety to give NiTi alloys photocatalytic properties.

\section{Conclusions}

We prepared photocatalytic $\mathrm{TiO}_{2}$ layers on NiTi alloy surfaces through a simple thermal oxidation process. The $\mathrm{TiO}_{2}$ layers were formed by heating NiTi alloys in air at temperatures above $723 \mathrm{~K}$, and the crystallinity of oxide varied from amorphous to rutile with the oxidation temperature. The prepared layers included small amounts of $\mathrm{Ni}$ in the topmost surface regions, in a $\mathrm{NiO}$ chemical state. The oxidized surface acted as a photocatalyst under UV light illumination, and its activity was higher than that of oxidized pure Ti substrates. The adhesion strength of the surface layer for a $723 \mathrm{~K}$ oxidation temperature was notably higher than that of $\mathrm{TiO}_{2}$ layers produced using a commercially available reagent. In conclusion, thermal oxidation using a temperature of $723 \mathrm{~K}$ is an excellent technique to produce photocatalytic surface layers having high adhesion strengths on NiTi alloy surfaces, and this technique is promising for use in medical practice.

\section{Acknowledgement}

The authors gratefully acknowledge Mr. M. Yamane from Kitami Institute of Technology for his support with XRD analysis, and also acknowledge Mr. W. Saito, a graduate student in our laboratory, for his help in XPS measurements. This work was supported by a Grant-in-aid for Scientific Research (C) (No. 24560841) from the Ministry of Education, Culture, Sports, Science and Technology (MEXT) of Japan.

\section{REFERENCES}

1) O. Fukushima, T. Yoneyama, H. Doi and T. Hanawa: Dent. Mater. J. 25 (2006) 151-160.

2) V. Sharma, R. Yngard and Y. Lin: Adv. Colloid Interface Sci. 145 (2009) 83-96.

3) T. Tanabe, N. Okitsu, A. Tachibana and K. Yamaguchi: Biomaterials 23 (2002) 817-825.

4) H. Sashiwa and S. Aiba: Prog. Polym. Sci. 29 (2004) 887-908

5) G. A. Sotiriou and S. E. Prasinis: Environ. Sci. Technol. 44 (2010) 5649-5654.

6) R. A. Ahmed, S. A. Fadl-allah, N. El-Bagoury and S. M. F. G. El-Rab: Appl. Surf. Sci. 292 (2014) 390-399.

7) A. K. Benabbou, Z. Derriche, C. Felix, P. Lejeune and C. Guillard: Appl. Catal. B 76 (2007) 257-263.

8) D. M. A. Alrousan, P. S. M. Dunlop, T. A. McMurray and J. A. Byrne: Water Res. 43 (2009) 47-54.

9) Y. Oka, W. Kim, T. Yoshida, H. Mouri, H. Urade, Y. Itoh and T. Kubo: Int. J. Biomed. Mater. Res. Part B: Appl. Biomater. 86B (2008) 530540.

10) P. Evans, T. English, D. Hammond, M. E. Pemble and D. W. Sheel: Appl. Catal. A 321 (2007) 140-146.

11) F. X. Ye, A. Ohmori and C.-J. Li: Surf. Coat. Technol. 184 (2004) 233238.

12) S. Takeda, S. Suzuki, H. Odaka and H. Hosono: Thin Solid Films 392 (2001) 338-344.

13) M. H. Wong, F. T. Cheng and H. C. Man: Appl. Surf. Sci. 253 (2007) 7527-7534.

14) D. Vojtěch, J. Fojt, L. Joska and P. Novak: Surf. Coat. Tech. 204 (2010) 3895-3901.

15) S. Wu, X. Liu, Y. L. Chan, J. P. Y. Ho, C. Y. Chung, P. K. Chu, C. L. Chu, K. W. K. Yeung, W. W. Lu, K. M. C. Cheung and K. D. K. Luk: J. Biomed. Mater. Res. 81A (2007) 948-955.

16) Y. W. Gu, B. Y. Tay, C. S. Lim and M. S. Yong: Appl. Surf. Sci. 252 (2005) 2038-2049.

17) Y. Wu, Y. He, T. Chen, W. Weng and H. Wan: Mater. Lett. 59 (2005) 3106-3108

18) K. T. Jacob, V. S. Saji and S. N. S. Reddy: J. Chem. Thermodyn. 39 (2007) 230-235.

19) G. S. Firstov, R. G. Vitchev, H. Kumar, B. Blanpain and J. V. Humbeeck: Biomaterials 23 (2002) 4863-4871.

20) N. Shevchenko, M.-T. Pham and M. F. Maitz: Appl. Surf. Sci. 235 (2004) 126-131.

21) C. H. Xu, X. Q. Ma, S. Q. Shi and C. H. Woo: Mater. Sci. Eng. A 371 (2004) 45-50.

22) T. Sreethawong, Y. Suzuki and S. Yoshikawa: Int. J. Hydrogen Energy 30 (2005) 1053-1062.

23) K. S. Kim, K. K. Jee, W. C. Kim, W. Y. Jang and S. H. Han: Mater. Sci. Eng. A 481-482 (2008) 658-661.

24) N. Ohtsu, T. Takahara, M. Hirano and H. Arai: Surf. Coat. Tech. 239 (2014) 185-190. 